\title{
GPU Scripting and Code Generation with PyCUDA
}

\author{
Andreas Klöckner* Nicolas Pinto ${ }^{\dagger} \quad$ Bryan Catanzaro $^{\ddagger}$ \\ Yunsup Lee ${ }^{\ddagger} \quad$ Paul Ivanov ${ }^{\S} \quad$ Ahmed Fasih
}

$23^{\text {rd }}$ April, 2013

High-level scripting languages are in many ways polar opposites to GPUs. GPUs are highly parallel, subject to hardware subtleties, and designed for maximum throughput, and they offer a tremendous advance in the performance achievable for a significant number of computational problems. On the other hand, scripting languages such as Python favor ease of use over computational speed and do not generally emphasize parallelism. PyCUDA is a package that attempts to join the two together. This chapter argues that in doing so, a programming environment is created that is greater than just the sum of its two parts.

We would like to note that nearly all of this chapter applies in unmodified form to PyOpenCL, a sister project of PyCUDA, whose goal it is to realize the same concepts as PyCUDA for OpenCL.

\footnotetext{
${ }^{*}$ Courant Institute of Mathematical Sciences, New York University, New York, NY 10012

${ }^{\dagger}$ Brain and Computer Sciences, Massachusetts Institute of Technology, Cambridge, MA 02139

${ }^{\ddagger}$ Electrical Engineering and Computer Sciences, University of California, Berkeley, CA 94720

${ }^{\S}$ Redwood Center for Theoretical Neuroscience, University of California, Berkeley, CA 94720

๑ Department of Electrical and Computer Engineering, Ohio State University, Columbus, OH 43210
} 


\section{Introduction, Problem Statement, and Context}

How are computational codes created? Their life cycle often begins with a quick proof of concept in a high-level language such as MATLAB or Python that aims to examine the suitability of the proposed method for the application problem at hand. Once this is established, the problem size is scaled up, and with it, the demands for execution speed grow. In principle, it is not desirable that the working proof-of-concept code would need to be changed merely to squeeze out better computational performance. Unfortunately, at present, very few workloads can be scaled in this fashion, so eventually the need arises to employ a language that produces efficient machine code.

The desire to make this transition as seamless as possible quite naturally leads to hybrid code, in which only those pieces requiring improved performance are changed, and the remainder is kept as-is. These approaches are not new (cf. e.g. MATLAB's Mex or Python's F2Py). Unfortunately, they are not as wide-spread as they could be because they come with a significant complexity burden. GPU computing adds a new facet to this issue, as GPU programs are always hybrids, even though NVIDIA's CUDA Runtime programming interface goes to considerable lengths to paper over this fact. GPU programs are thus very naturally split between performancehungry and performance-indifferent parts, usually along the same lines as the CPU-GPU boundary. This is fortunate, as one may easily substitute the performance-indifferent part of the hybrid, taking advantage of the already well-defined interface between the two. PyCUDA fits exactly into this niche and allows codes written in this high-level language to obtain GPU performance from within existing Python programs with a minimum of effort.

But in addition to a practical route to high performance for existing codes, PyCUDA also has much to offer to the seasoned GPU programmer creating full-scale, production codes. Many of these advanced benefits are described in detail in a recent manuscript Klöckner et al. [2009], which focuses on the capability of generating GPU code at run time and represents a more academic discussion of the software engineering aspects of GPU programming and what PyCUDA does to address them. This chapter on the other hand provides a hands-on perspective of the things one can do with PyCUDA, and how. 


\section{Core Method}

A common lament in the field of scientific computing concerns the everwidening gap between hypothetical machine capabilities and the effort an individual programmer is able to spend to move a computational application towards exploiting a machine to the full extent of its capability. GPUs obviously influence this balance, by increasing the maximum capability, by increasing the performance that is obtained with an "average" amount of effort, and by requiring a different set of skills to achieve genuinely high performance.

More sophisticated tools, compilers and libraries are generally hoped to level this field, enabling users to achieve good results even with modest investment. PyCUDA is a contribution to this discussion of tools for GPU computing. PyCUDA (and likewise its sister project PyOpenCL) has a two-fold aim. First, it aims to simplify the usage of existing basic concepts of CUDA C. Importantly, it does not attempt to change or reinvent the basic notions of GPU programming, but instead, as a foundation for further tools, just exposes them as-is through a carefully engineered interface. Key features of this interface include generous use of sensible defaults (all of which can be overridden), automatic error checking, and resource management. Second, and strictly on top of the first, basic layer, PyCUDA provides abstractions that support a number of very common usage patterns involving a GPU equivalent of NumPy [Oliphant, 2006] arrays.

As a particular consequence of the design choice to leave as much of the underlying concepts in place, device kernels occur in PyCUDA programs and PyOpenCL as simple strings. This is practical because Python allows socalled triple-quoted strings which may extend across line boundaries, thus appearing as contiguous code blocks without intervening string terminators or other traces of the host-level language. A different approach is pursued by the Clyther project 1 and similar efforts, which let the user write kernels in the host language and then translate them to the device language $(\mathrm{C} / \mathrm{C}++)$ in some manner. Each approach represents one end of a trade-off. It is obviously appealing to let the user program in one single language (the host language) rather than forcing her to master two separate ones. PyCUDA and PyOpenCL sacrifice this advantage in favor of passing on the concepts of the underlying programming system in as unmodified a manner as possible in order to provide direct applicability of existing CUDA/OpenCL

\footnotetext{
${ }^{1}$ http://clyther.sourceforge.net/
} 
documentation and to decrease the maintenance burden as the underlying system evolves. One additional factor entering the trade-off is the ease with which kernel code can be automatically generated (see Section 3.3.).

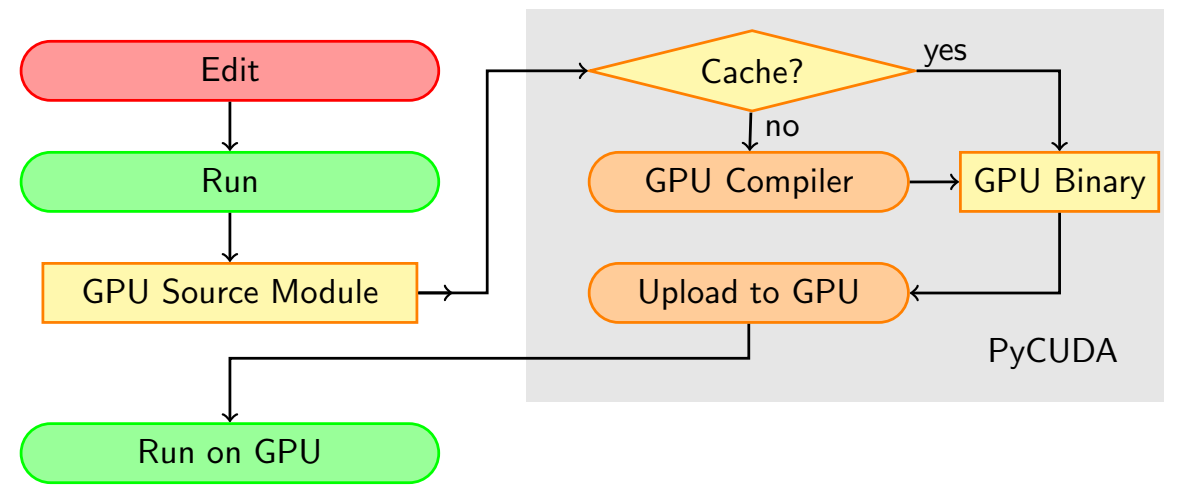

Figure 1: Workflow of PyCUDA GPU program compilation. PyCUDA aims to maintain a scripting-like "edit-run-repeat" style of working for the user. The compilation and caching operations in the gray box are performed without user involvement.

Programming in scripting languages (like Python) tends to be quite satisfying to the programmer because of near-immediate response during development, the possibility of interactive exploration, and good error reporting. Although PyCUDA gives the user access to a compiled language (CUDA C), it attempts to avoid the development iteration penalty commonly associated with compiled languages, instead retaining the satisfaction and immediacy of scripting. First of all, it makes invocations to the CUDA $\mathrm{C}$ compiler fast and transparent. To this end, it employs a compiler caching mechanism that is pictured in Figure 1. As it is likely that only one GPU kernel is being changed in each development iteration, the user only needs to wait for compilation of this one kernel. The loading of all other kernels in the code will be near-instantaneous thanks to PyCUDA's caching mechanism.

We have thus completed an initial description of PyCUDA's goals and usage patterns. The next section will, by means of a number of concrete examples of increasing complexity, show how common tasks can be accomplished in PyCUDA. Section 4 will then briefly reflect on what was shown, and we close in Section [6 with a few remarks and ideas for future work. 


\section{Algorithms, Implementations, and Evaluations}

In this section, we will take the reader on a brief journey through different aspects of programming GPUs using PyCUDA, starting with a basic hello-world example in Section 3.1, through more advanced aspects in the following sections.

\subsection{The Basics of GPU programming with PyCUDA}

Perhaps the simplest useful program that can be written using PyCUDA is shown in Listing 1, which we will discuss here step-by-step.

PyCUDA's interface to the 'nuts and bolts' of the CUDA programming system can be found in pycuda.driver and is imported here under the alias cuda. The module is called driver because it exposes the so-called "driver-level" programming interface of CUDA, which is more flexible than the more commonly used CUDA C "runtime-level" programming interface, and it has a few features that are not present in the runtime. (If a program uses the triple-angle-bracket syntax for kernel invocation, it is using the runtime interface.)

The next imported module, pycuda.autoinit, automatically picks a GPU to run on, based on availability and the number, if any, to which the CUDA_DEVICE environment variable is set. It also creates a GPU context for subsequent code to run in. Both the chosen device and the created context are available from pycuda.autoinit as importable symbols, if needed. The use of pycuda.autoinit is not compulsory-if needed, users can construct their own device-choice and context-creation methods using the facilities available in pycuda.driver.

The last import in this simple example is numpy, the Python array package. Since most GPU computations involve large arrays of data, PyCUDA integrates tightly with numpy.

After creating a $4 \times 4$ array of random single-precision floating point numbers on the CPU in the numpy array identified by the variable a, memory for a is allocated on the GPU as a_gpu. The object returned here is a DeviceAllocation object, whose lifetime is coupled to that of the GPU memory allocation, i.e. once the last reference to the DeviceAllocation disappears, the object becomes eligible for garbage collection, and once that happens, the GPU allocation also disappears. DeviceAllocation objects 


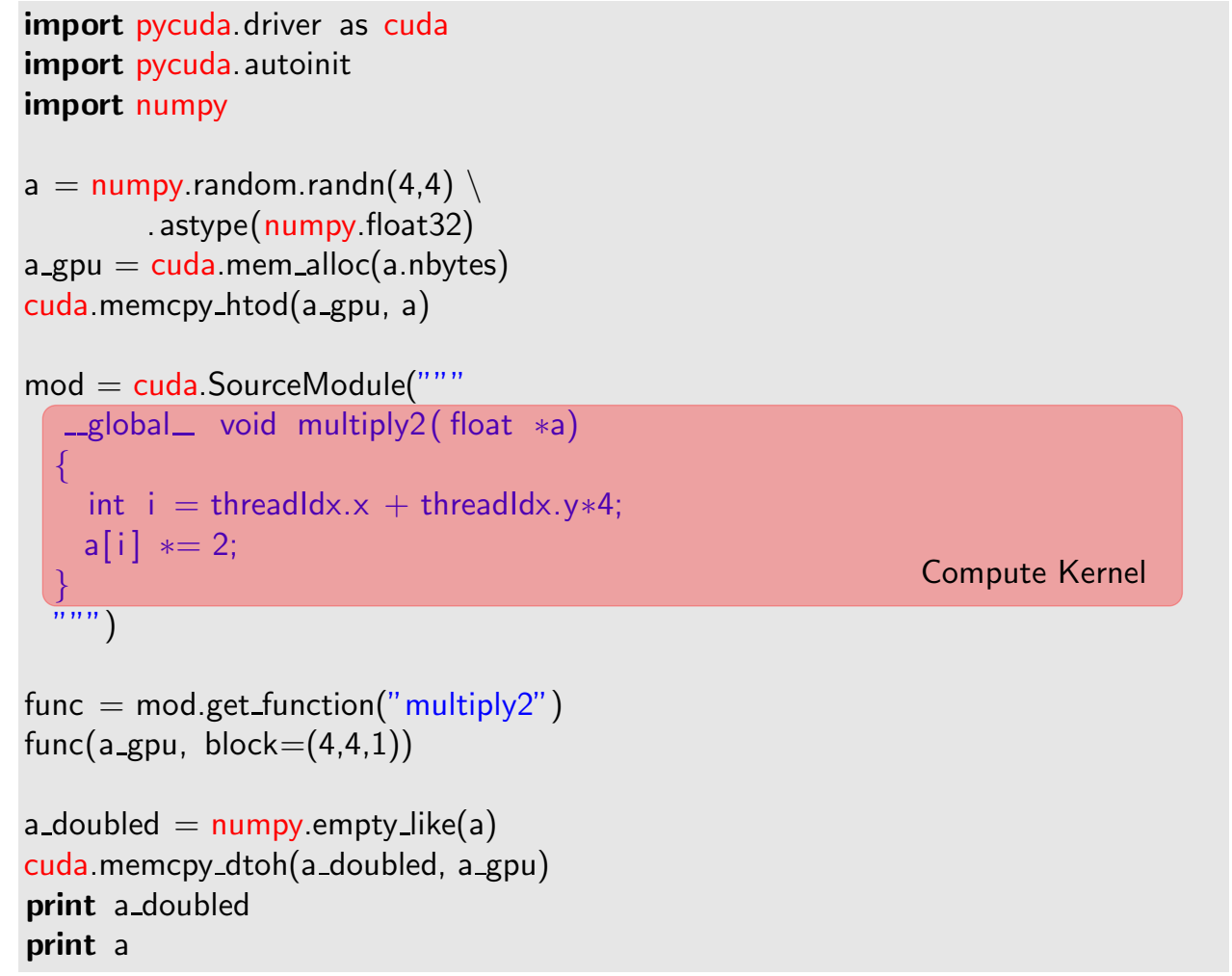

Listing 1: An example of the use of PyCUDA, showing the use of the SourceModule facility. This simple program uploads a $4 \times 4$ array of singleprecision floating point numbers, multiplies them by two on the GPU, and retrieves the result.

can also be cast to integer for pointer arithmetic. Once memory is allocated, the contents of a are copied from the host to the device (hence "htod"). Observe that no explicit error checking occurs at any stage of this program. If an error is encountered, a subclass of pycuda.driver.Error is raised as an exception.

Now that the data is prepared, the most interesting part of the program begins, in which the source code for the GPU kernel is passed to the constructor of SourceModule. At this point, the NVIDIA compiler is invoked, coupled with the caching mechanism as described in Section 2, at the end of which the user obtains a handle to a driver. SourceModule representing 
the binary code uploaded to the GPU. From this module handle, the user may then obtain a Kernel handle by means of the get_function method. Observe that $\mathrm{C}++$ name mangling 2 would generally make the symbol names passed to get_function complicated and non-human-readable. For this reason, PyCUDA automatically wraps the code passed to SourceModule in an extern " $\mathrm{C}$ " declaration. If the use of $\mathrm{C}++$ features is desired, the keyword argument no_extern_c may be passed to SourceModule to avoid this. In this case, any _-global_ entry points should be declared extern "C" by hand.

The Kernel handle, once it has been obtained, can then be called like any other function. Keyword arguments grid and block determine the size of the computational grid and the thread block size. DeviceAllocation instances may be passed directly to kernels, but other arguments incur the problem that PyCUDA knows nothing about their required type. There are two ways to address this: First, pass all such arguments as numpy sized scalars, such as numpy.float32(5.7) for single-precision floating point, or numpy. $\operatorname{intp}(\mathrm{p})$ for pointer-sized integers. Alternatively, one may use prepared kernel invocation, in which the user informs PyCUDA explicitly about the kernel's argument types. In this case, the invocation line would need to be changed as follows:

func. prepare("P", block=(4,4,1))

func. prepared_call $((1,1)$, a_gpu $)$

Note that the prepare method only needs to be called once. Its first argument represents the kernel's arguments as a type string as accepted by the struct module in Python standard library, e.g. "P" in the example specifies a single pointer argument. The prepared call styles also differs in that the thread block size is set at prepare time (but can be changed), and the grid dimension may be set with each call. Prepared kernel invocation is also slightly faster than the explicitly-sized invocation style.

In this example, the function of the GPU code itself is trivial-for a $4 \times 4$ block size and a single-element grid, each entry of the corresponding array on the GPU is multiplied by two. As the kernel is invoked, the kernel call enters the queue on the GPU, but, like in the CUDA "runtime" interface, the invocation returns immediately and does not wait for completion on the

\footnotetext{
2 "Name mangling" facilitates function overloading in $\mathrm{C}++$ and represents the encoding of signature information in the symbol name used for a function. Mangling methods vary by compiler and operating system ABI.
} 
GPU. An argument stream may be passed in either calling style to specify a Stream in which execution is to take place.

At the end of this simple example, memory is allocated on the CPU and results are transferred back, to be printed alongside the original array. Again, like in the CUDA runtime interface, all memcpy_* functions enqueue the transfer and wait until it completes. If this is not desired, separate memcpy_*_async functions exist.

\subsection{What Comes in the Box}

After the introduction to the basic method of programming GPUs with PyCUDA, this section seeks to make the reader aware of further built-in facilities aimed at making GPU programming easier.

\subsubsection{GPU Arrays}

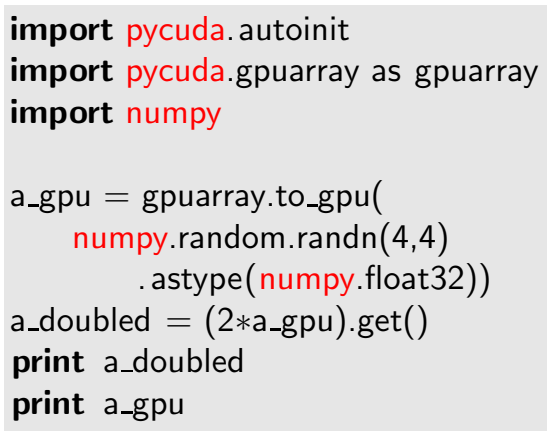

Listing 2: An example performing the same function as Listing 1, but using GPUArrays.

The example presented above, as an element-wise vector operation, represents not only an easy introduction, but also a common use case of GPU computations, perhaps in the form of an auxiliary step between other calculations. For this reason, PyCUDA supplies an array object, pycuda.gpuarray . GPUArray that shrinks the code of the example of Listing 1 to that of Listing 2, Just like numpy arrays, but unlike memory allocated using DeviceAllocation, GPUArrays know about their shapes and data types. They support all arithmetic operators and a number of methods and functions, all patterned after the corresponding functionality in numpy. In addition, many special 
functions are available in pycuda.cumath. Arrays of approximately uniformly distributed random numbers may be generated using functionality in pycuda. curandom.

\subsubsection{Complex Numbers on the GPU}

In addition to CUDA's built-in support for real numbers, PyCUDA adds seamless support for complex numbers. To enable this support, add the line "\#include <pycuda-complex.hpp>" to your kernel and declare complex numbers as pycuda: : complex<type $>$, where type may be, e.g., double or float. PyCUDA's GPUArrays also natively support operations on complex data types.

\subsubsection{Double Precision Textures}

Another area where PyCUDA improves on native CUDA is support for double precision in texture fetches. This is easiest to achieve when binding a GPUArray to a texture reference using the GPUArray's bind_to_texref_ext method, while specifying the allow_double_hack keyword argument as true. Within kernel code, one may then use the following pattern for texture access:

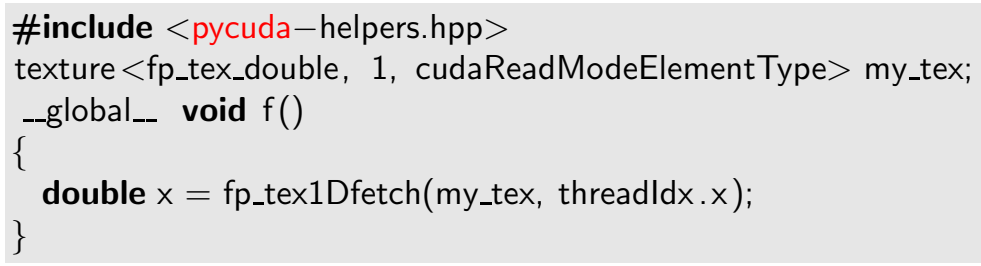

Observe in particular that the texture type was prefixed with fp_tex_ and the fetching function was prefixed with $\mathrm{fp}_{-}$.

\subsubsection{Efficient Evaluation of Element-wise Expressions}

Evaluating deeply nested expressions on GPUArray instances can be inefficient, because a new temporary is created for each intermediate result. Many programming languages offering user-definable abstract vector types have this problem, for example $\mathrm{C}++$. $\mathrm{C}++$ also offers a way of dealing with this particular issue in the form of expression templates 


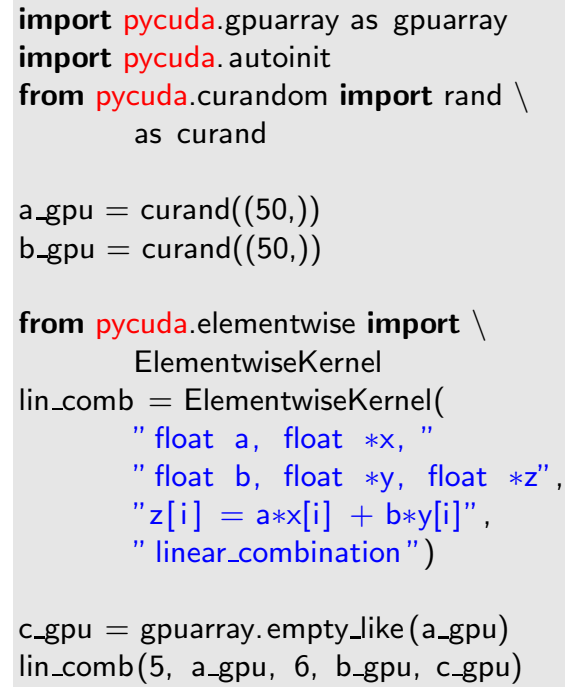

(a) An example of the use of the generic facility for element-wise operations.

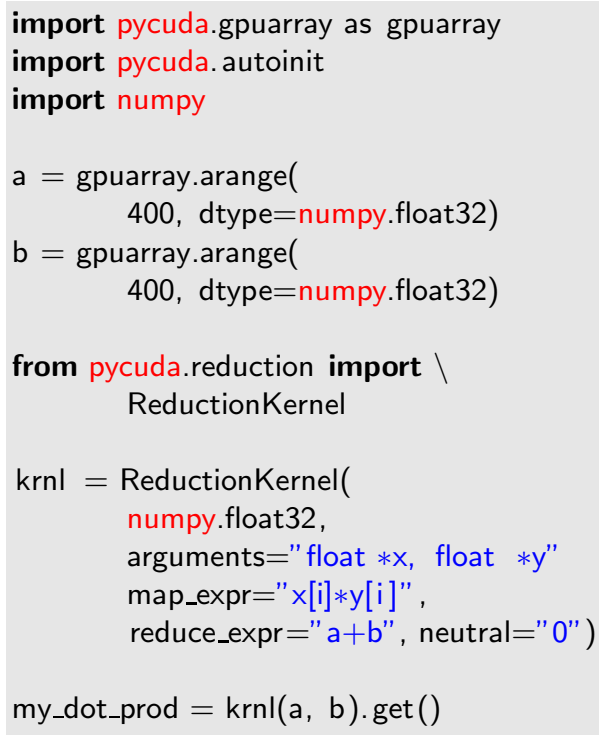

(b) An example of the use of the generic facility for reductive ("folding") operations.

Listing 3: Examples of high-level primitives for working with GPUArrays.

Veldhuizen and Jernigan, 1997]. While we acknowledge that this is certainly a matter of taste, we strongly prefer the striking simplicity of both use and implementation of PyCUDA's facility, portrayed in Listing 3(a), over the significant complexity of expression templates.

The functionality in the module pycuda. elementwise contains tools to help generate and invoke kernels that evaluate complicated expressions on one or several operands in a single pass. The instrumental part of the example is the invocation of the ElementwiseKernel constructor, in which the user provides both a C-style argument list and a statement (or semicolon-separated list of statements) to be executed for each value of $i$, which is used as a formal index variable running across each element of GPUArray instances passed to the ElementwiseKernel. All these instances are required to have the same length. 


\subsubsection{Map-Reduce}

In a similar spirit as the support for element-wise operations, PyCUDA has built-in functionality for tree-based reductions on the GPU. To make this even more useful, evaluating an element-wise expression ahead of reduction is also supported. The facility thereby becomes a simple implementation of the MapReduce procedure [Dean and Ghemawat, 2008].

Listing 3(b) shows the implementation of a dot product through an instantiation of the ReductionKernel class. The constructor signature starts with the specification of the result dtype, in this case numpy.float32. It then proceeds as the ElementwiseKernel above by allowing a $\mathrm{C}$ argument signature and an arbitrary $\mathrm{C}$ expression on whose results the final reduction will be performed. As above, the formal variable $i$ represents the index from which the input element should be read.

The reduction step is then specified by two further arguments, a reduction expression of two formal arguments $\mathrm{a}$ and $\mathrm{b}$ and an expression resulting in $\mathrm{a}$ neutral element with respect to the reduction expression 3 Once all of this information is specified, the resulting ReductionKernel may be called as above to perform the reduction.

Observe that the end result of calling the ReductionKernel instance is a GPUArray scalar still residing on the GPU. It can be brought to the CPU by a call to its get method, or used in-place on the GPU.

\subsubsection{Further Facilities}

In addition to elementwise operations and reductions, versions 2011.1 and newer of PyCUDA and PyOpenCL are able to assist the user with the implementation of GPU-based parallel prefix sums (also known as 'parallel scan'). Further, they provide a number of tools to the GPU implementer which we will mention here, but for whose detailed description we refer to the reference documentation.

The first of those is a key optimization for programs that allocate and deallocate GPU memory at a rapid rate. Since CUDA's memory allocation functions are relatively expensive operations, it becomes expedient to retain

\footnotetext{
3 "Neutral element" is mathematical terminology for an element that turns a binary operator into an identity map. For example, zero is the neutral element of addition, and one is the neutral element of multiplication.
} 


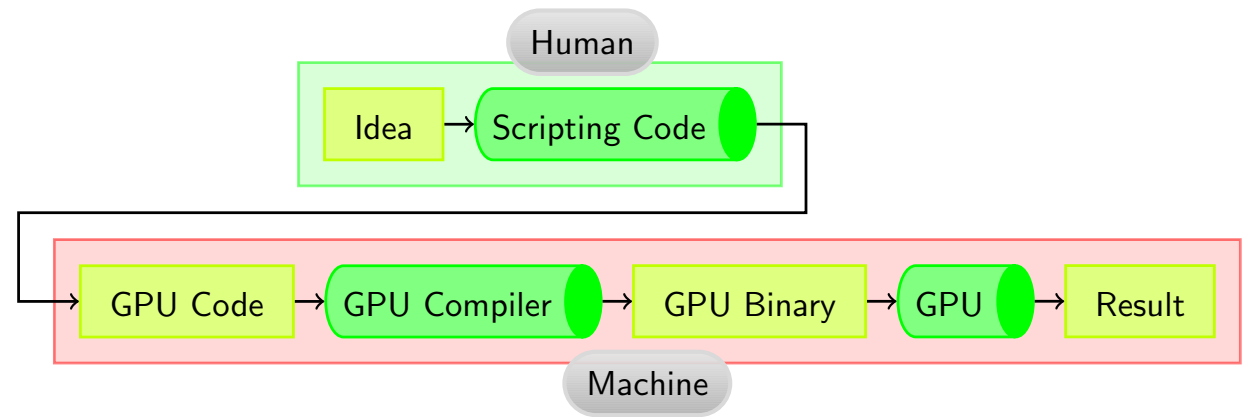

Figure 2: Operating principle of GPU code generation.

already allocated memory in a GPU computing process instead of freeing it. These retained blocks of memory may then be reused once a similarlysized block is requested afterwards. PyCUDA supports this through the use of memory pools. These pools integrate with GPUArrays, whose arithmetic operators are a good example of the need for repeatedly freed and allocated memory of recurring sizes. In addition, a memory pool implementation also exists for page-locked host memory.

Lastly, PyCUDA comes with a conjugate-gradient-based Krylov solver for large, sparse linear systems and an implementation of sparse matrices on the GPU, following [Bell and Garland, 2009]. These GPU-based sparse matrices integrate directly with sparse matrix support in the SciPy package [Jones et al., 2001-] and provide computational performance similar to that of NVIDIA's Cusp [Bell and Garland, 2010] library.

\subsection{Code Generation: Benefits and Usage}

As early as 30 years ago, the Lisp community observed that code is data, and that using code itself as the object of computation can be greatly beneficial. One key benefit of PyCUDA and PyOpenCL is that they make run-time code generation ("RTCG") almost trivial. Figure 2 clarifies the workflow used in RTCG.

This section is devoted to describing a number of issues that are commonly faced when programming a GPU. In each case, we point out how a GPU RTCG strategy can be used to address these issues. 


\subsubsection{Automated Tuning}

During the creation of a GPU program, it is natural for the programmer to come up with a number of variants of a given code, each of which will be observed to have certain properties regarding data layout and computation speed. The conventional approach to code tuning then calls for the fastest variant to survive, while the others will be discarded. This is not necessarily a desirable course of action, as information is lost. Instead, it seems more appropriate to retain as many of these variants as is practical, assuming that they hold at least some promise. Further, each variant may have a number of tunable parameters, such as loop lengths, block sizes, etc. Retaining variant information permits choosing the best one from a reasonably sized pool of candidates in an automated fashion, guided by some metric such as execution speed. This is the basic premise of automated tuning, which is trivially enabled by GPU RTCG. Further, automated tuning is not just enabled by RTCG, it is enabled at the right time-namely at run timewhen complete information is available. If desired, the reader may find a few illustrative examples of the use of automated tuning in Klöckner et al., 2009].

\subsubsection{The Cost of Flexibility}

Flexibility is commonly seen as a desirable feature of a computer code. It should then be realized that flexibility comes at a cost: Constants get replaced by variables, formerly fixed loop trip counts become variable, and quite generally a compiler has less knowledge available, making its optimizer

less effective. The process of removing this sort of flexibility by hard-coding such information into the program, therefore, is generally frowned upon. However, with the availability of run-time code generation, information can be inserted into the source of the program just in time, leading to an optimal combination of flexibility and execution speed.

\subsubsection{High-Performance Abstractions}

Nearly all computer programs are built in 'layers', where each individual layer solves a certain subproblem and presents a more abstract, 'higherlevel' interface to higher layers. This is good engineering practice, as it allows partitioning a big problem into many smaller ones, and it enables 
reuse of engineering effort. In some cases, such abstractions can be made uneconomical by coding circumstance, namely when customization applies to the contents of an inner loop. Many solutions exist to this problem, ranging from function pointers to $\mathrm{C}++$ templates, each with unique disadvantages [Klöckner et al., 2009]. Once RTCG is available, this problem also disappears as appropriate code can be generated whenever a different requirement arises.

\subsubsection{Generating Code}

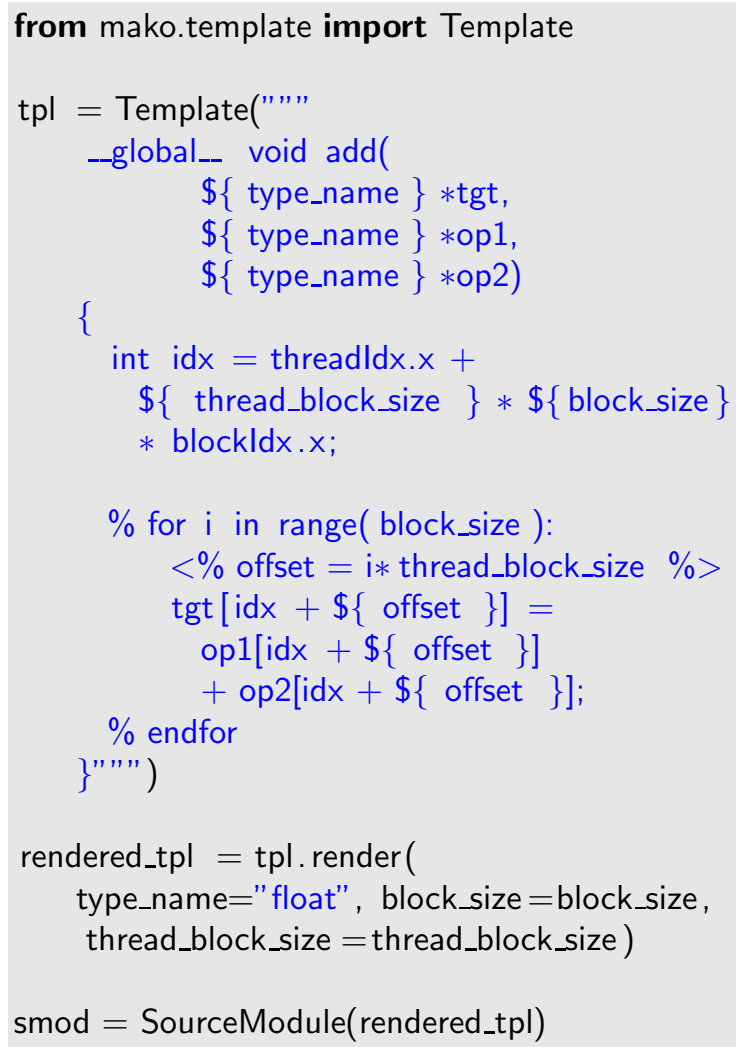

Listing 4: Run-Time Code Generation (RTCG) with PyCUDA using a templating engine. The Example generates a piece of CUDA $\mathrm{C}$ from a textual template implementing an unrolled version of vector addition, using the Mako engine this instance. Full context for the example can be found in the PyCUDA source tree as examples/demo_meta_template.py. 
We now turn to how a user might go about exploiting run-time code generation with PyCUDA. Since PyCUDA can natively process CUDA C code, the objective is the generation of such code. PyCUDA makes no assumptions about the origins of the code it processes, which allows the logic involved in the generation to be designed to match the needs of the application. There are, however, a few suggested ways of generating code that we have found to cover a variety of needs.

Code generation can (and in many cases should) be seen as a text processing task. Since one is not limited in the choice of tools with which to perform this sort of generation, code generation typically makes use of existing text processing tools. Generation logic itself can thus be simple and generally responds favorably to complexity growth.

Textual keyword replacement. This simple technique performs the equivalent of search-and-replace on source code. It suffices for a surprisingly large range of use cases, such as the substitution of types and constants into source code at run time. Its technological reach is increased by combining it with $\mathrm{C}$ preprocessor macros. Further contributing to its attractiveness, Python's standard library can perform keyword substitution without relying on external software.

Textual Templating. For code generation applications where control flow and conditionals are required, but where all code variants are textually related, the use of a so-called templating engine, commonly used for the generation of web pages, offers a natural escalation of the capabilities of keyword substitution. Many templating engines (and correspondingly, templating languages) exist. Listing 4 demonstrates the use of the MakcBaver [2010] engine for the generation of a simple, partially unrolled vector addition code.

In addition to these methods, the authors' codepy package also supports code generation from abstract syntax trees (ASTs), however this use is somewhat cumbersome and discouraged in all but the most demanding cases.

\section{Evaluation}

While it is difficult to obtain quantifiable data on GPU programmer productivity and how the use of the PyCUDA and PyOpenCL packages affects

it, one of the main measures of the success of any open-source package is 
the size and vitality of the community that uses and develops it.

As such, we believe that the wide existing user base of PyCUDA and PyOpenCL represents compelling evidence that this programming model as well as its concrete implementations are a significant improvement in the way programmers interact with GPUs, thereby serving as an important step toward bringing GPU computing to the mainstream.

One central point for user collaboration is each package's wiki at http://wiki.tiker.net/PyCuda and http://wiki.tiker.net/PyOpenCL. In a user-editable fashion, each functions as a central collection point for installation instructions on a variety of operating systems, frequently asked questions, and code examples.

In addition, a number of packages have been released by community members that build on top of PyCUDA and PyOpenCL, including

PyFFT (by Bogdan Opanchuk) An FFT package for both PyCUDA and PyOpenCL. Also a nice example of cross-CUDA/OpenCL code generation. http://pypi.python.org/pypi/pyfft.

Scikits.CUDA (by Lev Givon and collaborators) Offers wrappers of the CUBLAS, CUFFT and CULA packages for numerical computation on CUDA. http://pypi.python.org/pypi/scikits.cuda.

Atomic Hedgehog (by Cyrus Omar) Offers a higher-level programming interface for PyOpenCL. http://ahh.bitbucket.org/.

For a listing of projects that use PyCUDA or PyOpenCL in production, see [Klöckner et al., 2009] and http://wiki.tiker.net/PyCuda/ShowCase.

\section{Availability}

PyCUDA is available from http://mathema.tician.de/software/pycuda, and PyOpenCL is available from http://mathema.tician.de/software/pyopencl. Both are distributed under the liberal MIT open-source software license.

Full documentation is available online at http://documen.tician.de/pycuda and http://documen.tician.de/pyopencl, respectively, and both packages include numerous examples and automated tests. The packages support all platforms on which Python and CUDA and/or OpenCL are available. 


\section{$6 \quad$ Future Directions}

PyCUDA and PyOpenCL, as open-source projects, thrive on user feedback, particularly feedback regarding limitations, bugs, or missing features that users encounter. For example, much of PyCUDA's initial feature set emerged in support of an effort to bring discontinuous Galerkin methods onto the GPU, as discussed elsewhere in this volume.

As part of this continuing improvement process, we have identified a number of core areas in which we see potential for future work. Much work has recently been done to make PyCUDA easier to install. Part of this effort was the elimination of the Boost $\mathrm{C}++$ library as an explicit dependency, which was released as part of PyCUDA version 0.94 and PyOpenCL 0.92 in September of 2010. We are also working on easing integration with existing CUDA tools such as CUBLAS, CUFFT and Thrust Hoberock and Bell, 2010]. Integration and ease of migration between PyCUDA and PyOpenCL is another key feature that we would like to facilitate. We are further planning to improve GPUArray's capability of dealing with slices of multidimensional arrays. Finally, we are planning on improving support for automated tuning in PyCUDA and PyOpenCL by providing search algorithms on top of user-supplied search space descriptions and speeding up exploration of compilation-bound searches by exploiting all available CPU cores.

We hope that this chapter has managed to encourage you, the reader, to try what we believe is a very productive, full-featured GPU programming environment. We look forward to your questions and comments on our mailing lists.

\section{Acknowledgments}

AK's research was partially funded by AFOSR under contract number FA955007-1-0422, through the AFOSR/NSSEFF Program Award FA9550-10-1-0180 and also under contract DEFG0288ER25053 by the Department of Energy. The opinions expressed are the views of the authors. They do not necessarily reflect the official position of the funding agencies. 


\section{References}

Mike Bayer. Mako: A super-fast templating language, 2010. http://makotemplates.org, Version 0.3.6.

N. Bell and M. Garland. Implementing sparse matrix-vector multiplication on throughput-oriented processors. In SC '09: Proceedings of the 2009 ACM/IEEE conference on Supercomputing, New York, NY, USA, 2009. ACM.

Nathan Bell and Michael Garland. Cusp: Generic parallel algorithms for sparse matrix and graph computations, 2010. http://cusp-library.googlecode.com, Version 0.1.0.

J. Dean and S. Ghemawat. MapReduce: Simplified data processing on large clusters. Communications of the ACM, 51(1):107-113, 2008.

Jared Hoberock and Nathan Bell. Thrust: A parallel template library, 2010. http://www.meganewtons.com/, Version 1.3.0.

Eric Jones, Travis Oliphant, Pearu Peterson, et al. SciPy: Open source scientific tools for Python, 2001-. URL http://www.scipy.org/.

Andreas Klöckner, Nicolas Pinto, Yunsup Lee, Bryan C. Catanzaro, Paul Ivanov, and Ahmed Fasih. PyCUDA: GPU Run-Time Code Generation for High-Performance Computing. Technical report, Brown University, Providence, RI, USA, November 2009. submitted, http://arxiv.org/abs/0911.3456.

T. Oliphant. Guide to NumPy. Trelgol Publishing, Spanish Fork, UT, July 2006.

T. L. Veldhuizen and M. E. Jernigan. Will C ++ be faster than Fortran? In Proceedings of the 1st International Scientific Computing in ObjectOriented Parallel Environments (ISCOPE'97), Lecture Notes in Computer Science. Springer-Verlag, 1997. 